\title{
ESSAI DE CLASSIFICATION DES COCCIDIES \\ DE LA FAMILLE DES DIPLOSPORIDAE LÉGER, 1911
}

\author{
Par A. HENRY et Ch. LEBLOIS
}

La littérature zoologique et médicale s'est enrichie dans ees dernières années d'un grand nombre de publications ayant trait à des coccidies de l'homme et des carnivores, et classées, jusqu'à présent, dans le genre Isospora.

On sait que ce genre avait été établi par A. Schneider, en 1881, pour un sporozoaire, trouvé chez une petite limace indéterminée, et dont l'étude, très imparfaite, n'a jamais pu être reprise.

La description, très sommaire, dit, au sujet des sporozoïtes : " corpuscules des spores en nombre indéfini " et encore : " corpuscules falciformes assez nombreux ". Malgré ces indications, l'Isospora de Schneider devint une coccidie disporocystée tétrazoïque à la suite des paraphrases de Léger (1898), puis de Laveran (1898) qui, interprétant les figures de l'auteur, estimèrent que les spores contenaient quatre sporozoites; à tort ou à raison, leur opinion a généralement prévalu.

En tenant pour exacte cette manière de voir, il n'en reste pas moins que le fait de comporter deux fois quatre sporozoïtes, constitue un caractère si banal qu'il devient impossible de lui conserver une simple valeur générique; le nombre et la diversité des espèces qu'on est obligé de ranger sous son chef se sont tellement accrus que le groupement ainsi constitué a bien acquis la valeur d'une famille, la famille des Diplosporidæ Léger, 1911, dans laquelle nécessité s'impose d'établir des coupures.

Dès 1902, Ed. Sergent avait amorcé ce sectionnement. Il partageait les espèces d'Isospora connues, à cette époque, en deux catégories : une première à sporocystes piriformes avec $I$. rara, I. lacazei, I. camillerii, I. mesnili, et une seconde à sporocystes ovoïdes comprenant $I$. laverani, I. lieberkühni.

Revenant sur cette séparation, Mesnil, en 1914, indique que peut-être il y aurait lieu " de scinder le genre Isospora (= Diplospora) en deux, suivant que les sporocystes sont piriformes (G.Isospora s. s.) ou ovoïdes; pour ces derniers on adopterait le genre Hyaloklossia Labbé (espèce type Hyaloklossia lieberkühni) avec une diagnose modifiée ".

AnNales de Parasitologie, $\mathrm{T}$. IV, $\mathrm{x}^{\circ} 1 .-1^{e r}$ janvier 1926. p. 22-28. 
Ainsi, d'après Mesnil, les coccidies di-tétrazoïques qui intéressent actuellement médecins et vétérinaires et dont aucune ne possède de spores en poire, devraient être rangées dans le genre Hyaloklossia.

La note de Mesnil a dû sans doute échapper à la grande majorité des auteurs comme elle avait échappé à nous-mêmes ; en tous cas, il n'apparaît pas, jusqu'ici, qu'on en ait fait état.

Et cependant le caractère sur lequel s'appuie cette première subdivision, c'est-à-dire la forme des sporocystes, mérite réellement d'être retenu.

Pour parachever la subdivision entreprise, il nous semble, en outre, qu'un second ordre de caractère pourrait encore intervenir, à savoir : la disposition des sporozoïtes dans le sporocyste.

En utilisant les renseignements fournis sur ce point par les auteurs, nous croyons pouvoir distinguer, d'une part, un genre Diplospora distinct d'Isospora, et, d'autre part, un nouveau genre Lucetina (1), comprenant, entre autres, toutes les coccidies di-tétrazoïques de l'Homme et des Carnivores, distinct d'Hyaloklossia.

La famille des Diplosporidæ Léger, 1911, groupant toutes les coccidies à deux sporocystes tétrazoïques, et ayant pour type le genre Diplospora A. Labbé, 1893, renfermerait donc, pour l'instant, selon nous, quatre genres qui peuvent se distinguer de la façon suivante:

Sporocystes inéquipolaires (piriformes) $\ldots \ldots \ldots \ldots \ldots \ldots \ldots \ldots, 1$

Sporocystes équipolaires (sphèriques, ellipsoïdes ou fusiformes).. 2

Sporozoïtes dans le même sens ................. Isospora.

Sporozoïtes deux par deux, tête-bêche........... Diplospora.

2

Sporozoïtes dans le mème sens................. Lucelina.

Sporozoïtes croisés deux par deux ............... Hyaloklossia.

\section{A. Genre Isospora A. Schneider, 1881}

Isospora L. Léger, 1898, emend. ; Isospora Laveran, 1898, pro parte; Isospora auct. pro parte.

Diplosporidæx à oocyste sphérique ou légèrement ovalaire à paroi épaisse à double contour ; sporocystes piriformes ; pas de reliquat cystal ; sporozoïtes assez longs repliés ou enroulés parallèlement en spirale ; reliquat sporal représenté par quelques rares granulations ou nul. Mollusques (Limax); reptiles (sauriens).

(1) Dédié à la mémoire d'Adrien Lucet, ancien vétérinaire à Courtenay (Loiret) et assistant de Pathologie comparée au Muséum national d'Histoire naturelle, en raison de ses nombreux travaux sur les coccidies. 
Type : Isospora rara A. Schneider, 1881; d'une petite limace noire.

Tel que nous le concevons, le genre Isospora comprendrait encore :

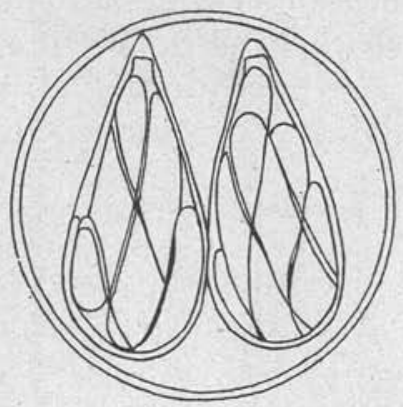

FıG. 1. - Isospora rara, d'après Schneider. Grossissement inconnu.

Isospora camillerii (Hagenmuller, 1898) ; Diplospora camillerii (Hgm., 1898) ; Isospora camillerii (E. Sergent, 1902). De Gongylus ocellatus.

Isospora mesnili E. Sergent, 1902. De Chamœeleo vulgaris.

\section{B. Genre Diplospora A. Labbé, 1893}

Isospora Laveran, 1898, pro parte.

Diplosporidæ à oocyste sphérique ou légèrement ovalaire, à paroi épaisse à double contour ; sporocystes piriformes ; pas de reliquat

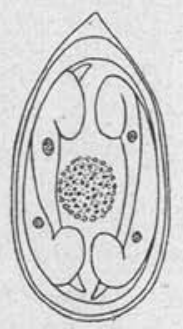

Fig. 2. - Diplospora lacazei, un sporocyste mûr, d'après A. Labbé. Grossissement approximatif : 1500 .

cystal ; sporozoïtes emboîtés deux à deux tête-bêcho; un reliquat sporal; sporulation exogène. Epithélium intestinal des oiseaux (passériformes).

Type : Diplospora avium (Rivolta, 1878) ; [Psorospermium avium Riv., 1878 ; Isospora avium Railliet, 1885; Diplospora lacazei 
A. Labbé, 1893 (Isospora lacazei Lav., 1898); Diplospora rivoltæ A. Labbé, 1893 (non Coccidium rivolta Grassi, 1882 ; non Coccidium rivoltæ, Harz, 1887) ; Diplospora passerum (Nils Sjöbring, 1897) (Isospora passerum N. Sj., 1897)]. D'un très grand nombre d'espèces de passériformes.

Diplospora sp. De Meleagris gallopavo (Th. Smith, 1917).

\section{c. Genre Lucetina Henry et Leblois, 1925}

Isospora auct. pro parte ; Hyaloklossia Mesnil, 1914, pro parte.

Diplosporida à oocyste sphérique, ellipsoïde ou en bissac ; sporocystes sphériques ou ellipsoídes; pas de reliquat cystal; spo-

A

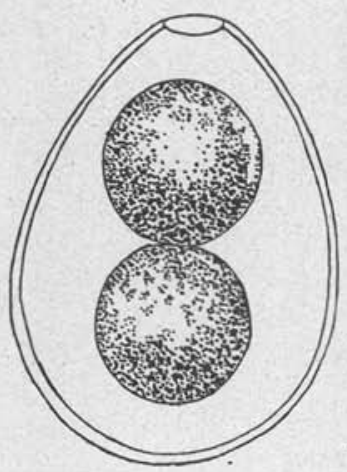

$B$

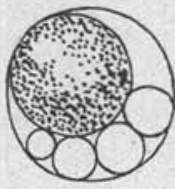

$C$.

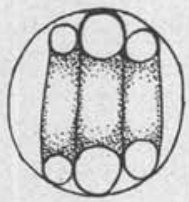

Fig. 3. - Lucetina rivoltai, d'après Grassi ; A, oocyste avec sporoblastes ; B et C, sporocystes avec sporozoïtes ; en C, le reliquat n'a pas été indiqué. Grossissement approximatif : 1500 .

rozoïtes indépendants, placés parallèlement et dans le même sens ; un reliquat sporal. Tissu épithélial ou subépithélial : carnivores, homme, reptiles.

Type : Lucetina rivoltai (Grassi, 1881) (Coccidium rivolta Grassi, 1881, non Coccidium rivoltx Harz, 1887, non Diplospora rivoltai A. Labbé, 1893 ; Coccidium rivoltai, Railliet, 1893) ; Felis domestica, Canis familiaris.

Dans ce genre, on peut encore classer :

Lucetina felis (Wenyon, 1923) (Isospora cati Marotel, 1922 ; Isospora felis Wenyon, 1923). De Felis domestica, Canis familiaris, Felis leo (J. Möller, 1923). 
Lucetina belli (Wenyon, 1923) (Isospora belli Wenyon, 1923; Isospora hominis Dobell, 1919)(1). De Homo sapiens.

Lucetina canivelocis (Weidemann, 1915) (Coccidium bigeminum var, canivelocis Weid., 1915 ; Isospora canivecolis (lapsus) Wenyon, 1923). De Vulpes velox.

Lucetina sp., Coccidies rondes de Basset (1909). De Canis familiaris, Putorius furo.

Lucetina laverani (Hagenmuller, 1898) (Diplospora laverani Hgm., 1898 ; Isospora laverani E. Sergent, 1902). De Coelopeltis lacertina.
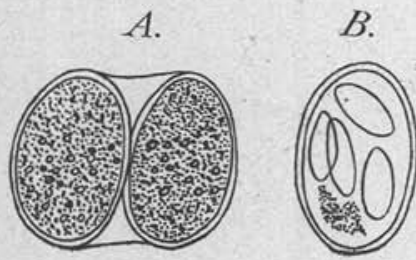

Fıg. 4. - Lucetina bigemina des villosités intestinales du chien, d'après Railliet ; A, un oocyste avec deux sporocystes; B, un sporocyste avec sporozoïtes et reliquat. Grossissement : 1500 .
FIG. 5. - Lucetina bigemina, sporocyste mûr dans les excréments d'un chien, d'après Nieschulz. Grossissement : 1500 .

Lucetina bigemina (Stiles, 1891) (Coccidium bigeminum Stiles, 1891 ; C. bigeminum var. canis Railliet et Lucet, 1891; Isospora sp.? Nieschulz, 1925). De Canis familiaris.

Lucetina cati (Railliet et Lucet, 1891) (Coccidium bigeminum var. cati R. et L., 1891) ; Isospora bigeminum Nieschulz, 1925). De Felis domestica.

Lucetina putorii (R. et L., 1891) (Coccidium bigeminum var. putorii R. et L., 1891). De Mustela putorius.

Lucetina hominis (R. et L., 1891) (Coccidium bigeminum var. hominis R. et L., 1891 ; Isospora hominis Wenyon, 1923). De Homo sapiens.

Lucetina sp. (Isospora sp. Labbé, 1899 et Coccidium sp. Labbé, 1899 : Coccidium Grassi, 1881). De Coronella austriaca Laur.

(1) Dans un travail récent, R. Pons (Bull. Soc. path. exol., 1925, n॰ 7, p. 578) signale dans cette espèce des sporozoïtes à disposition alternée, mais animés, sur de nombreux exemplaires, de mouvements " assez importants pour changer la position primitive, relative des sporozoïtes $n$. 


\section{Genre Hyaloklossia A. Labbé, 1896}

\section{(Laveran et Mesnil, 1902, emend.)}

Isospora Lav. et Mesnil, 1902 ; Hyaloklossia Mesnil, 1914, pro parte.

Diplosporidæ à membrane mince, se rupturant facilement ; sporocystes ovalaires ou fusiformes; pas de reliquat cystal; quatre sporozoïtes (parfois deux seulement, par avortement) croisés deux à deux ; reliquat sporal sphérique volumineux. Batraciens.

Type : Hyaloklossia lieberkühni A. Labbé (1894) (Klossia lieberkühni Labbé, 1894; Isospora lieberkuhni Laveran et Mesnil, 1902). Dans les reins de Rana esculenta et $R$. temporaria.

Peut-être faut-il rapporter au genre $\mathrm{Hya}$ loklossia les Coccidium disporées tétrazoïques signalées par Grassi (1881) dans l'intestin d'un crapaud et que Labbé a déjà rattaché au genre Diplospora (1896) ou au genre Isospora (1899).

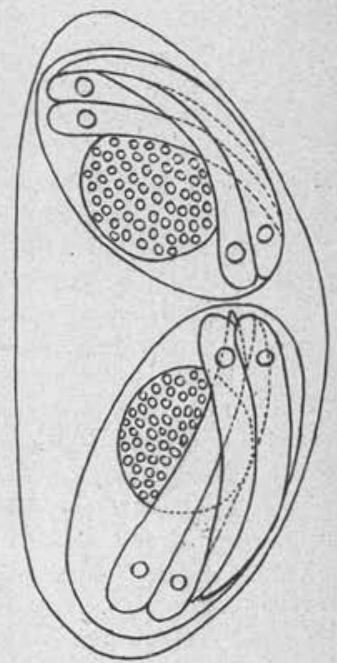

Fig. 6. - Hyaloklossia lieberkühni, d'a près Laveran et Mesnil. Grossissement : 1500 .

\section{Bibliographie}

BASset (J.). - La coccidiose intestinale, maladie des jeunes animaux. Bull. Soc. Cent. Méd. Vét., LXIII, 1909, p. 463.

Dobell (C.). - A revision of the Coccidia parasitic in Man. Parasitology, XI, 1919, p. 147 ,

Grassi (B.). - Intorno ad alcuni protisti endoparassitici ed appartenenti alle classi dei Flagellati, Lobosi, Sporozoi e Ciliali. Atti Soc. Ital. Sci. Nat., XXIV, 1881 , p. 135.

- Sur quelques protistes endoparasites appartenant aux classes des Flagellata, Lobosa, Sporozoa et Ciliata. Arch, ital, Biol., II, 1882, p. 402.

Hagensuler (P.). - Sur une nouvelle Coccidie parasite du Gongylus ocellatus. C. R. Soc. Biol., L, 1898, p. 73.

- Sur une nouvelle Coccidie diplosporée (Diplospora Laverani $\mathrm{Hgm}$.), parasite d'un Ophidien. C. R. Soc. Biol., L, 1898, p. 309.

Henry (A.) et Leblois (Ch.). - Essai de classification des Coccidies disporocystées tétrazoïques. C. R. Ac. Sc., CLXXX, n 9, 1925, p. 700.

Labbé (A.). - Sur les Coccidies des oiseaux. C. R. Ac. Sc., CXVI, no 23, 1893, p. 1300.

- Recherches zoologiques, cytologiques et biologiques sur les Coccidies. Aich. Zool. exp., IV (3e série), 1896. 
Laвве́ (A.). - Sporozoa. Das Tierreich, Berlin, 1899, p. 517.

Laveran (A.). - Sur les modes de reproduction d'Isospora Lacazei. C. R. Soc. Biol. L, 1898 , p. 1139

Lavelan (A.) et Mesniz (F.). - Sur la Coccidie trouvée dans le rein de la Rana esculenta et sur l'infection générale qu'elle produit. C. R. Ac. Sc., CXXXV, 1902 , p. 82.

LÉger (L.). - Essai sur la classification des Coccidies et description de quelques espèces nouvelles ou peu connues. Bull. Mus. Marseille, I, fasc. I, 1898, p. 80 .

- Caryospora simplex, Coccidie monosporée et la classification des Coccidies. Arch. f. Protislenk., XXII, 1911, p. 71-88.

Marotel. (G.). - Sur une nouvelle Coccidie du chat. Bull. Soc. Sci. Vétér. Lyon, 1921 , p. 86.

Messil. (F.). - Bull. Inst. Pasteur, XII, 1914, p. 838.

Mölt.er (J.). - Kokzidien bei den Säugetieren des Zoologischen Gartens zu Berlin. Inaug. Diss. Doct. Med. Vet., Berlin, 1923.

Nizschulz (O.). - Zur Verbreitung von Isospora. Infektionen bei Hunden und Katsen in den Niederlanden. Centralbl. f. Balit. Par. und Infekt., Erste Abt., Originale, XCIV, H. 2, 1925, p. 137.

Pospiech (W.). - Untersuchungen über den mikroskopischen Nachweis von Darmparasiten im Kot von Fleischfressern, mit besonderer Berücksichtigung der Kokzidien. Veterinärmed. Inaug. Diss., München, 1919.

Railliet (A.) et Lucet (A.). - Note sur quelques espèces de Coccidies encore peu étudiées. Bull. Soc. Zool. France, XVI, 1\$91, p. 249.

Schneider (Aimé). - Sur les Psorospermies oviformes ou Coccidies. Espèces nouvelles ou peu connues. Arch. Zool. exp., IX, 1881, p. 401.

Sergent (Ed.). - Sur une Coccidie nouvelle, parasite du caméléon vulgaire. $C$. $R$. Soc. Biol., LIV, 1902, p. 1260.

Sıöbring (N.). - Beiträge zur Kenntnis einiger Protozoen. Centralbl. f. Bakt., Originale, XXII, 1897, p. 675.

Sмrтн (Th.). - Journ. of experim. Med., v. 25, 1917, p. 405. (D'après Bull. Inst. Pasteur, XVl, 1918, p. 71).

Stıles (Ch.-W.). - Note préliminaire sur quelques parasites. Bull. Soc. Zool. France, 1891, p. 163.

Weidemann (F.-D.). - Coccidium bigeminum Stiles in Swift Foxes (Habitat Western U. S.). Journ. Comp. Path. and Therap., XXVIII, 1915, p. 320.

Wenyon (C.-M.). - Coccidiosis of Cats and Dogs and the Status of the Isospora of Man. Annals Trop. Med. and Par., XVII, 1923, p. 231.

Wetzel. (R.). - Ein Beitrag zur Coccidiose der Katze. Deulsche Tier. Woch. 33 Jahrg., n० 7,1925 , p. 97.

ZAPFE (H.). - Zur Kenntnis der Kokzidiose des Hundes. Inaug. Diss. Berlin, 1923.

Laboraloire de Parasilologie de lEcole vétérinaire d'Alforl. 